\title{
Analysis of Dynamics of Boundary Shape Perturbation of a Rotating Elastoplastic Radially Inhomogeneous Plane Circular Disk: Analytical Approach
}

\author{
Dmytro M. Lila ${ }^{1}$, A. A. Martynyuk ${ }^{2}$ \\ ${ }^{1}$ Cherkasy National Bohdan Khmelnytsky University, Cherkasy, Ukraine \\ ${ }^{2}$ Stability of Processes Department, S. P. Timoshenko Institute of Mechanics, \\ National Academy of Sciences of Ukraine, Kyiv, Ukraine \\ Email: dim_1@ukr.net
}

Received March 5, 2012; revised March 26, 2012; accepted April 3, 2012

\begin{abstract}
For a rotating inhomogeneous circular disk a way of calculating dynamics of boundary shape perturbation and failure of bearing capacity is proposed in terms of small parameter method. Characteristic equation of plastic zone critical radius is obtained as a first approximation. A formula of critical angular velocity is derived which determines the stability loss of the disc according to the self-balanced form. Efficiency of the proposed method is shown by an illustrative example considered in Section 7. Values of critical angular velocity of rotation are found numerically for different parameters of the disc.
\end{abstract}

Keywords: Axisymmetric Elastoplastic Problem; Method of Boundary Shape Perturbation; Rotating Inhomogeneous Circular Disc; Stability Loss; Failure of Bearing Capacity; Critical Angular Velocity

\section{Introduction}

The failure of bearing capacity of a quickly rotating elastic disc [1-3] overloaded by centrifugal dilating forces is associated with the dynamics of its boundary shape perturbation [4]. After the disc takes up new balanced shape due to considerable growth of plastic zones [5] the instability [6] develops torrentially enough with the increase of rotation velocity [7]. This is stipulated by the response of the internal points to the disc contour reshaping and outrunning growth of variable radius of the perturbed elastoplastic boundary as compared with the variation of its current radius for a stable disc $[8,9]$.

To study the stability loss and velocity dynamics of a rotating disc the perturbation method can be applied [10-12]. In the analysis of plane stress strain state this method was employed to obtain approximate critical values of the plastic zone dimensions and angular velocity of continuous homogeneous circular discs $[13,14]$, ring-shaped discs [15] including those loaded along the contour by additional radial forces [16], stepped discs and some arbitrary profile discs [17] as well as simplest inhomogeneous discs. This proves efficiency of the analytical method of boundary shape perturbation (with the use of simplest numerical procedures at certain stage) which reduces essentially the amount of calculations and at the same time facilitates fruitful application of various numerical techniques [18-20] for stability and strength calculation of turbine and other massive discs.

Meanwhile, there is still an open problem of establishing by the small parameter method the relationship between the value of boundary shape perturbation, plastic zone radius and rotation velocity of unstable con- tinuous inhomogeneous circular disc corresponding to the indicated state of perturbed elastoplastic boundary. This is the subject of our present investigation.

\section{Statement of the Problem}

We consider a disc D consisting of two homogeneous and isotropic plane discs D1 and D2. Continuous circular disc D1 possesses radius $a$ which coincides with the internal radius of the ring-shaped circular disc D2. The external radius of disc D2 equals to $b$. Discs D1 and D2 made from different materials are rigidly connected into one disc $\mathrm{D}$ along the circumference $r=a$. We designate by $\sigma_{s 1}$ the material yield limit of disc D1, $E_{1}$ is the elasticity modulus, $\gamma_{1}$ is the density and $v_{1}$ is the Poisson coefficient. The corresponding material parameters of disc D2 are designated by $\sigma_{s 2}, E_{2}, \gamma_{2}$ and $v_{2}$ respectively. It is assumed that constant angular rotation velocity $\omega$ of disc $\mathrm{D}$ is higher than its critical velocity $\omega_{*}$. This means the presence of perturbation of the initial contour circumference $r=b$, perturbation of the current 
radius of elastoplastic boundary $r=r_{0}$ and, in general, perturbation of stress strain state of the whole (unstable) disc.

We focus our attention on the self-balanced form of the stability loss of disc D which is little different from the circular form. The disc boundary equation up to the first order infinitesimal is presented in the form

$$
r=b+d \cos n \theta, \quad d=\text { const }, \quad n \geq 2,
$$

or

$$
\rho=1+\delta \cos n \theta
$$

where $\rho=r / b$ is a dimensionless current radius, $\delta$ is a small parameter, $n \in \mathbb{N}, \theta$ is a polar angle. On this basis, let us determine critical values $r=r_{0}^{*}$ and $\omega=\omega^{*}$ which accompany reaching of the above mentioned circumference $r=h$ by the perturbed elastoplastic boundary in the elastic zone of disc D. The critical values corresponding to reaching of the disc edge by the elastoplastic boundary, i.e. its contact with curve (1), should be specially calculated. We recall that for solution of these problems it is necessary, first of all, to analytically establish the condition of contact of the elastoplastic boundary and a circumference of given radius, i.e. to construct characteristic equation with the parameter $\delta$ with respect to $r_{0}$ having solved first the system of linear equations

$$
\begin{aligned}
& \sigma_{r r}+\frac{\mathrm{d} \sigma_{r r}^{0}}{\mathrm{~d} r} u=0 \text { for } r=b, \\
& \sigma_{r \theta}-\frac{\sigma_{\theta \theta}^{0}-\sigma_{r r}^{0}}{b} \frac{\mathrm{d} u}{\mathrm{~d} \theta}=0 \text { for } r=b, \\
& \sigma_{r r}=0 \text { for } r=r_{0}, \\
& \sigma_{r \theta}=0 \text { for } r=r_{0}, \\
& \sigma_{\theta \theta}+\frac{\mathrm{d} \sigma_{\theta \theta}^{0}}{\mathrm{~d} r} u=0 \text { for } r=r_{0}
\end{aligned}
$$

with respect to $u\left(r_{0}\right)$ and arbitrary constants found in the expressions for stress and displacement components $\sigma_{r r}, \sigma_{r \theta}, \sigma_{\theta \theta}$ and $u$ determining perturbed stress strain state of the rotating disc D. The above mentioned linearized perturbations of the first order of smallness satisfy differential balance equations of plane problem and partial differential equations of relationship between stresses and displacements [1]. Unperturbed stress state (designated by the upper index 0 ) is determined by ordinary differential equations of quasistatic equilibrium and constraint equations in the elastic zone or by the yield Saint-Venant condition [5] in the plastic zone.

In view of the instability development mechanism of the inhomogeneous disc under consideration the stated problem will be solved for each of the four cases: (a) D1peD2e (Figure 1(a)); (s2) D1pD2pe (Figure 1(b)); (b)

\section{D1eD2pe (Figure 1(c)); (c) D1peD2pe (Figure 1(d)).}

\section{Solution in the Case D1peD2e}

In order to use boundary and conjugation conditions

$$
\begin{aligned}
& \sigma_{\rho \rho}^{\prime e}+A_{1} u^{\prime e}=0 \text { for } \rho=1, \\
& \sigma_{\rho \theta}^{\prime e}-A_{2} \frac{d u^{\prime e}}{d \theta}=0 \text { for } \rho=1, \\
& \sigma_{\rho \rho}^{\prime e}=0 \text { for } \rho=\beta_{0}, \\
& \sigma_{\rho \theta}^{\prime e}=0 \text { for } \rho=\beta_{0}, \\
& \sigma_{\theta \theta}^{\prime e}+A_{3} u^{\prime e}=0 \text { for } \rho=\beta_{0},
\end{aligned}
$$

for perturbations of the first order of smallness $\sigma_{\rho \rho}^{\prime e}$, $\sigma_{\theta \theta}^{\prime e}$ and $\sigma_{\rho \theta}^{\prime e}$ of radial, contact and tangential stresses related to the yield limit $\sigma_{s 2}$ we recall that in D2e (Figure 1(a))

$$
\begin{aligned}
\sigma_{\rho \rho}^{\prime e}= & {\left[a_{I}(\beta, \rho) a_{2}+a_{I I}(\beta, \rho) a_{1}\right.} \\
& \left.+a_{I I I}(\beta, \rho) b_{2}+a_{I V}(\beta, \rho) b_{1}\right] \cos n \theta, \\
\sigma_{\rho \theta}^{\prime e}= & {\left[c_{I}(\beta, \rho) a_{2}+c_{I I}(\beta, \rho) a_{1}\right.} \\
& \left.+c_{I I I}(\beta, \rho) b_{2}+c_{I V}(\beta, \rho) b_{1}\right] \sin n \theta,
\end{aligned}
$$

and in D1e

$$
\begin{aligned}
\sigma_{\rho \rho}^{\prime e}= & {\left[a_{I}\left(\frac{\beta_{0}}{\beta}, \frac{\rho}{\beta}\right) a_{1}+a_{I I}\left(\frac{\beta_{0}}{\beta}, \frac{\rho}{\beta}\right) a_{0}\right.} \\
& \left.+a_{I I I}\left(\frac{\beta_{0}}{\beta}, \frac{\rho}{\beta}\right) b_{1}+a_{I V}\left(\frac{\beta_{0}}{\beta}, \frac{\rho}{\beta}\right) b_{0}\right] \cos n \theta, \\
\sigma_{\theta \theta}^{\prime e}= & {\left[b_{I}\left(\frac{\beta_{0}}{\beta}, \frac{\rho}{\beta}\right) a_{1}+b_{I I}\left(\frac{\beta_{0}}{\beta}, \frac{\rho}{\beta}\right) a_{0}\right.} \\
& \left.+b_{I I I}\left(\frac{\beta_{0}}{\beta}, \frac{\rho}{\beta}\right) b_{1}+b_{I V}\left(\frac{\beta_{0}}{\beta}, \frac{\rho}{\beta}\right) b_{0}\right] \cos n \theta, \\
\sigma_{\rho \theta}^{\prime e}= & {\left[c_{I}\left(\frac{\beta_{0}}{\beta}, \frac{\rho}{\beta}\right) a_{1}+c_{I I}\left(\frac{\beta_{0}}{\beta}, \frac{\rho}{\beta}\right) a_{0}\right.} \\
& \left.+c_{I I I}\left(\frac{\beta_{0}}{\beta}, \frac{\rho}{\beta}\right) b_{1}+c_{I V}\left(\frac{\beta_{0}}{\beta}, \frac{\rho}{\beta}\right) b_{0}\right] \sin n \theta,
\end{aligned}
$$

and, besides,

$$
\begin{aligned}
& a_{0}=q_{1}^{\prime} a_{2}+q_{2}^{\prime} a_{1}+q_{3}^{\prime} b_{2}+q_{4}^{\prime} b_{1}, \\
& b_{0}=q_{5}^{\prime} a_{2}+q_{6}^{\prime} a_{1}+q_{7}^{\prime} b_{2}+q_{8}^{\prime} b_{1} .
\end{aligned}
$$

Here $\beta_{0}=r_{01} / b, \beta=a / b, a_{2}, a_{1}, b_{2}$ and $b_{1}$ are indefinite coefficients and $q_{1}^{\prime}, \cdots, q_{8}^{\prime}$ are the coefficients expressed via $n, \beta_{0}, \beta, v_{1}, v_{2}$ and $\varepsilon=E_{2} / E_{1}$; $a_{I}(\cdot), \cdots, a_{I V}(\cdot), \quad b_{I}(\cdot), \cdots, b_{I V}(\cdot), c_{I}(\cdot), \cdots, c_{I V}(\cdot)$ are known functions [16]. Moreover, 


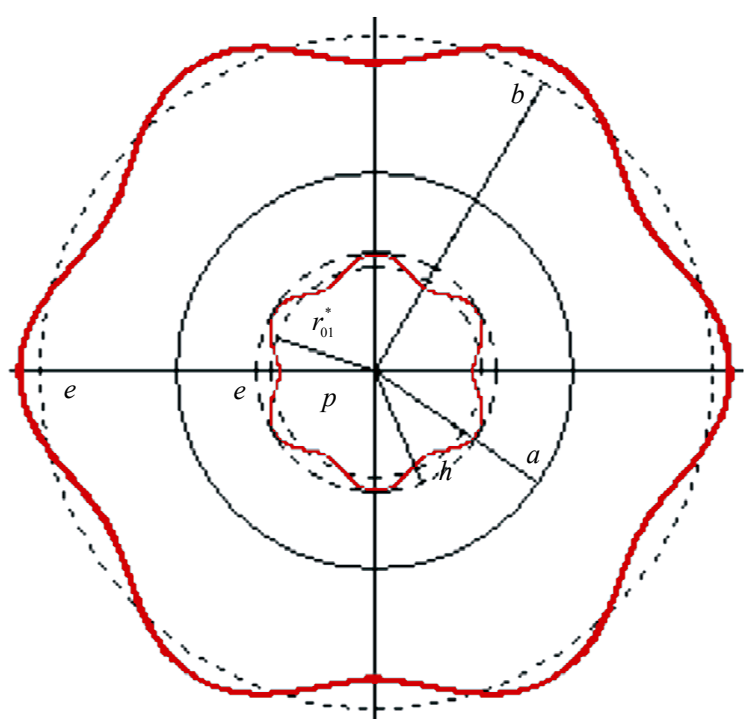

(a)

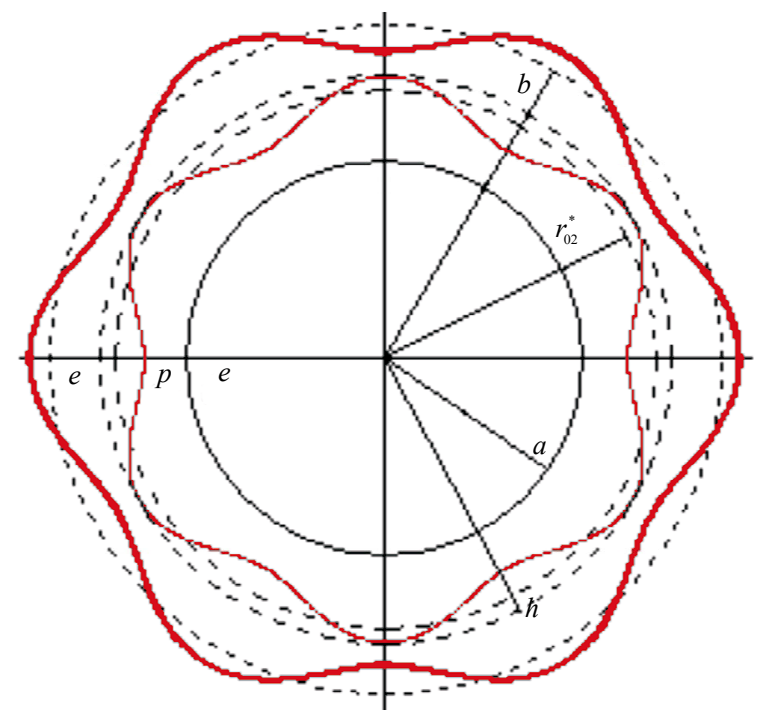

(c)

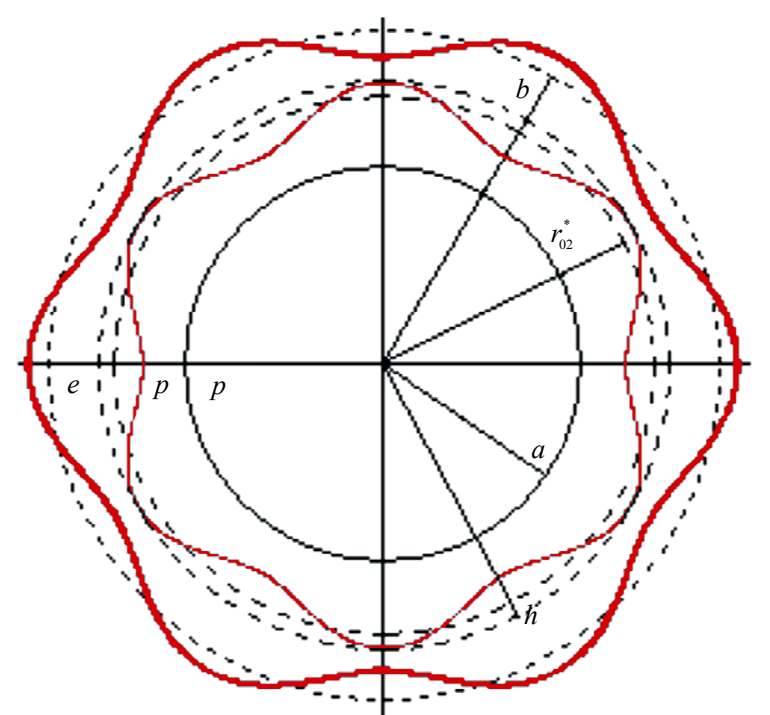

(b)

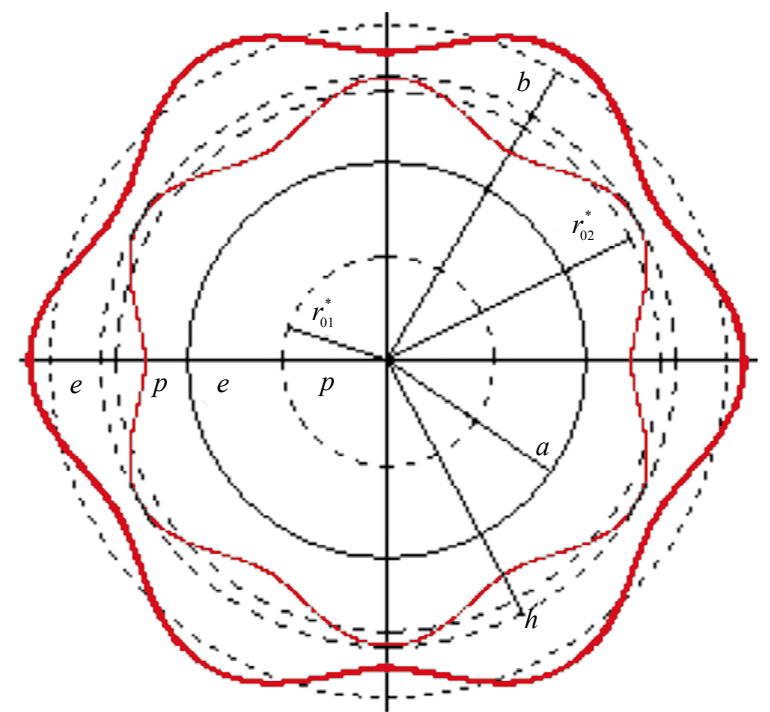

(d)

Figure 1. Perturbed elastoplastic boundary reaching given circumference $r=h$.

$$
\begin{aligned}
& A_{1}=2 C^{\prime}-6\left(v_{2}+3\right) x, A_{2}=A_{1}+24 x, \\
& A_{3}=-8 \beta_{0} \Gamma\left(3 v_{1}+1\right) x
\end{aligned}
$$

where

$$
\begin{aligned}
C^{\prime}= & \left\{s+\left[m+\Gamma\left(3 v_{1}+1\right)\left(2-\beta^{-2} \beta_{0}^{2}\right) \beta_{0}^{2}\right] x\right\}\left\{1-\beta^{-2}\right\}^{-1}, \\
x= & \omega^{2} /\left(24 q_{2}^{2}\right)=-s\left\{1+\beta^{2}+(\varepsilon+k)\left(1-\beta^{2}\right)\right\} \\
& \cdot\left\{\left(1+\beta^{2}\right)\left[m+\Gamma\left(3 v_{1}+1\right)\left(2-\beta^{-2} \beta_{0}^{2}\right) \beta_{0}^{2}\right]+\left(1-\beta^{2}\right)\right. \\
& \left.\cdot\left[l+\Gamma\left(3 v_{1}+1\right)\left\{2(\varepsilon+k)+\beta^{-2}(\varepsilon-k) \beta_{0}^{2}\right\} \beta_{0}^{2}\right]\right\}^{-1}, \\
s= & \sigma_{s 1} / \sigma_{s 2}, \Gamma=\gamma_{1} / \gamma_{2}, q_{2}=b^{-1} \sqrt{\sigma_{s 2} / \gamma_{2}}, k=v_{2}-\varepsilon v_{1},
\end{aligned}
$$

$l=$

$-3\left\{v_{2}+3+\beta^{2}\left\{\Gamma\left[\varepsilon\left(3 v_{1}+1\right)+k\left(v_{1}+3\right)\right]-\left(3 v_{2}+1\right)\right\}\right\}$, $m=-3\left\{v_{2}+3+\beta^{2}\left[\Gamma\left(v_{1}+3\right)-\left(v_{2}+3\right)\right]\right\}$.

For $\rho=1$ perturbation of the first order of smallness of the radial displacement related to $b$ is known from (1)

$$
u^{\prime e}(1)=\cos n \theta
$$

Therefore,

$$
\frac{\mathrm{d} u^{\prime e}(1)}{\mathrm{d} \theta}=-n \sin n \theta .
$$


Conditions (2)-(6) in the extended form [16] are

$$
\begin{aligned}
& a_{2}+A_{1}=0, \\
& b_{2}+n A_{2}=0, \\
& q_{1}^{\prime} a_{2}+q_{2}^{\prime} a_{1}+q_{3}^{\prime} b_{2}+q_{4}^{\prime} b_{1}=0, \\
& q_{5}^{\prime} a_{2}+q_{6}^{\prime} a_{1}+q_{7}^{\prime} b_{2}+q_{8}^{\prime} b_{1}=0, \\
& \left(w_{1} a_{2}+w_{2} a_{1}+w_{3} b_{2}+w_{4} b_{1}\right) \cos n \theta \\
& +A_{3} u^{\prime e}\left(\beta_{0}\right)=0,
\end{aligned}
$$

where

$$
\begin{aligned}
& w_{1}=b_{I I}(\zeta, \zeta) q_{1}^{\prime}+b_{I V}(\zeta, \zeta) q_{5}^{\prime}, \\
& w_{2}=b_{I}(\zeta, \zeta)+b_{I I}(\zeta, \zeta) q_{2}^{\prime}+b_{I V}(\zeta, \zeta) q_{6}^{\prime}, \\
& w_{3}=b_{I I}(\zeta, \zeta) q_{3}^{\prime}+b_{I V}(\zeta, \zeta) q_{7}^{\prime}, \\
& w_{4}=b_{I I I}(\zeta, \zeta)+b_{I I}(\zeta, \zeta) q_{4}^{\prime}+b_{I V}(\zeta, \zeta) q_{8}^{\prime}, \\
& \zeta=\beta_{0} / \beta .
\end{aligned}
$$

Hence,

$$
u^{\prime e}\left(\beta_{0}\right)=U\left(\beta_{0}\right) \cos n \theta,
$$

where

$$
U\left(\beta_{0}\right)=-\left(w_{1} a_{2}+w_{2} a_{1}+w_{3} b_{2}+w_{4} b_{1}\right) / A_{3},
$$

and, besides,

$$
\begin{aligned}
& a_{2}=-A_{1}, b_{2}=-n A_{2}, \\
& a_{1}=\left\{q_{4}^{\prime}\left(q_{5}^{\prime} a_{2}+q_{7}^{\prime} b_{2}\right)-q_{8}^{\prime}\left(q_{1}^{\prime} a_{2}+q_{3}^{\prime} b_{2}\right)\right\}\left\{q_{2}^{\prime} q_{8}^{\prime}-q_{4}^{\prime} q_{6}^{\prime}\right\}^{-1}, \\
& b_{1}=\left\{q_{6}^{\prime}\left(q_{1}^{\prime} a_{2}+q_{3}^{\prime} b_{2}\right)-q_{2}^{\prime}\left(q_{5}^{\prime} a_{2}+q_{7}^{\prime} b_{2}\right)\right\}\left\{q_{2}^{\prime} q_{8}^{\prime}-q_{4}^{\prime} q_{6}^{\prime}\right\}^{-1} .
\end{aligned}
$$

As a consequence, the characteristic equation with respect to the plastic zone radius corresponding to the moment of contact of the perturbed elastoplastic boundary and the mentioned circumference $\rho=h / b=\alpha$ becomes

$$
\alpha-\beta_{0}-\delta\left|U\left(\beta_{0}\right)\right|=0 .
$$

The critical value of angular velocity $\omega^{*}$ corresponding to the critical value of radius of the plastic domain $\beta_{0}=\beta_{0}^{*}, \beta_{0}^{*} \in\left[\beta_{0 *}, \alpha\right] \quad\left(\beta_{0 *}\right.$ is a critical radius of the plastic zone D1p for which the disc loses its stability) is obtained in terms of (7).

\section{Solution in the Case D1pD2pe}

Now, in contrast to Section 3, the elastic domain is homogeneous and represents zone D2e (Figure 1(b)), therefore $[14,15]$

$$
\begin{aligned}
& \sigma_{\rho \rho}^{\prime e}= \\
& {\left[n A \rho^{n-2}+n B \rho^{-n-2}+(n-2) C \rho^{n}+(n+2) D \rho^{-n}\right] \cos n \theta,} \\
& \sigma_{\theta \theta}^{\prime e}= \\
& {\left[-n A \rho^{n-2}-n B \rho^{-n-2}-(n+2) C \rho^{n}-(n-2) D \rho^{-n}\right] \cos n \theta,}
\end{aligned}
$$

$$
\sigma_{\rho \theta}^{\prime e}=\left[-n A \rho^{n-2}+n B \rho^{-n-2}-n C \rho^{n}+n D \rho^{-n}\right] \sin n \theta,
$$

where $A, B, C$ and $D$ are indefinite coefficients. Correlations (7) are written as

$$
\begin{aligned}
& A_{1}=2 C^{\prime}-6\left(v_{2}+3\right) x, A_{2}=A_{1}+24 x, \\
& A_{3}=-2 C^{\prime} \beta_{0}^{-3}-6 \beta_{0}\left(3 v_{2}+1\right) x,
\end{aligned}
$$

where

$$
\begin{aligned}
& C^{\prime}=\left\{1-3\left[v_{2}+3-\left(3 v_{2}+1\right) \beta_{0}^{2}\right] x\right\}\left\{1+\beta_{0}^{-2}\right\}^{-1}, \\
& x=\left\{2+\beta \beta_{0}^{-1}(s-1)\left(1+\beta_{0}^{2}\right)\right\} \\
& \cdot\left\{3\left[v_{2}+3-\left(3 v_{2}+1\right) \beta_{0}^{2}\right]\left(1-\beta_{0}^{2}\right)\right. \\
& \left.+\left[3\left(v_{2}+3\right)-\left(3 v_{2}+1\right) \beta_{0}^{2}+8 \beta^{3} \beta_{0}^{-1}(\Gamma-1)\right]\left(1+\beta_{0}^{2}\right)\right\}^{-1}, \\
& \beta_{0}=r_{02} / b .
\end{aligned}
$$

Therefore, the system of equations for determination of $u^{\prime e}\left(\beta_{0}\right)$ has the form

$$
\begin{aligned}
& n A+n B+(n-2) C+(n+2) D+A_{1}=0, \\
& -A+B-C+D+A_{2}=0, \\
& n \beta_{0}^{n-2} A+n \beta_{0}^{-n-2} B+(n-2) \beta_{0}^{n} C+(n+2) \beta_{0}^{-n} D=0, \\
& -\beta_{0}^{n-2} A+\beta_{0}^{-n-2} B-\beta_{0}^{n} C+\beta_{0}^{-n} D=0, \\
& \left(-n \beta_{0}^{n-2} A-n \beta_{0}^{-n-2} B-(n+2) \beta_{0}^{n} C-(n-2) \beta_{0}^{-n} D\right) \cos n \theta \\
& +A_{3} u^{\prime e}\left(\beta_{0}\right)=0 .
\end{aligned}
$$

Hence,

$$
u^{\prime e}\left(\beta_{0}\right)=U\left(\beta_{0}\right) \cos n \theta,
$$

where

$$
\begin{aligned}
& U\left(\beta_{0}\right) \\
& =\left(n A \beta_{0}^{n-2}+n B \beta_{0}^{-n-2}+(n+2) C \beta_{0}^{n}+(n-2) D \beta_{0}^{-n}\right) / A_{3},
\end{aligned}
$$

and, moreover,

$$
\begin{aligned}
& A=\left(A_{1}\left[n \beta_{0}^{2}-\beta_{0}^{-2 n}-(n-1)\right]\right. \\
& \left.-A_{2}\left[n^{2} \beta_{0}^{2}+(n-2) \beta_{0}^{-2 n}-(n-1)(n+2)\right]\right) /(2 N), \\
& B=\left(A_{1}\left[n \beta_{0}^{2}+\beta_{0}^{2 n}-(n+1)\right]\right. \\
& \left.-A_{2}\left[-n^{2} \beta_{0}^{2}+(n+2) \beta_{0}^{2 n}+(n+1)(n-2)\right]\right) /(2 N), \\
& C=\left(A_{1}\left[n \beta_{0}^{-2}+\beta_{0}^{-2 n}-(n+1)\right]\right. \\
& \left.-A_{2} n\left[(n+2) \beta_{0}^{-2}-\beta_{0}^{-2 n}-(n+1)\right]\right) /(2 N), \\
& D=\left(A_{1}\left[n \beta_{0}^{-2}-\beta_{0}^{2 n}-(n-1)\right]\right. \\
& \left.-A_{2} n\left[-(n-2) \beta_{0}^{-2}-\beta_{0}^{2 n}+(n-1)\right]\right) /(2 N),
\end{aligned}
$$




$$
N=2\left(n^{2}-1\right)-n^{2}\left(\beta_{0}^{2}+\beta_{0}^{-2}\right)+\left(\beta_{0}^{2 n}+\beta_{0}^{-2 n}\right) .
$$

Thus, characteristic Equation (8) is constructed.

The characteristic equation with a parameter with respect to critical radius of the plastic domain which reached the external edge of the disc D reads [9]

$$
1-\beta_{0}-\delta\left(1-U\left(\beta_{0}\right)\right)=0
$$

where $U\left(\beta_{0}\right) \leq 0$ for $\delta \geq 0$.

\section{Solution in the Case D1eD2pe}

Having compared stress states (unperturbed and perturbed) of the disc D in cases (s1) and (b) (Figure 1(c)) of instability development we conclude about the necessity to preserve here all calculations of Section 4 having changed the expression for $x=\omega^{2} /\left(24 q_{2}^{2}\right)$

$$
\begin{aligned}
& x=\left\{\varepsilon+k+0,5 \beta(1-(\varepsilon+k))\left(1+\beta_{0}^{2}\right) \beta_{0}^{-1}\right\} \\
& \left\{(\varepsilon+k)\left[3\left(v_{2}+3\right)-\left(3 v_{2}+1\right) \times\left(2-\beta_{0}^{2}\right) \beta_{0}^{2}\right]\right. \\
& \left.-\beta^{3}\left[4(\varepsilon+k)-3 \Gamma \varepsilon\left(1-v_{1}\right)\right]\left(1+\beta_{0}^{2}\right) \beta_{0}^{-1}\right\}^{-1}, \\
& \beta_{0}=r_{02} / b .
\end{aligned}
$$

\section{Solution in the Case D1peD2pe}

In order to follow the perturbation dynamics of elastoplastic boundary between D2p and D2e (Figure 1(d)) we will take into account (see Section 4) the fact that

$$
\begin{aligned}
& C^{\prime}=\left\{1-3\left[v_{2}+3-\left(3 v_{2}+1\right) \beta_{0}^{2}\right] x\right\}\left\{1+\beta_{0}^{-2}\right\}^{-1}, \\
& \beta_{0}=r_{02} / b
\end{aligned}
$$

$$
x=\frac{1-s(\varepsilon+k)}{\Gamma\left\{\left(3 v_{1}+1\right)\left[2(\varepsilon+k)+(\varepsilon-k) \beta^{-2} \beta_{01}^{2}\right] \beta_{01}^{2}-3 \beta^{2}\left[\varepsilon\left(3 v_{1}+1\right)+k\left(v_{1}+3\right)\right]\right\}},
$$

where

$$
\begin{aligned}
& \beta_{01}^{2}=\left(-c_{2} \pm \sqrt{c_{2}^{2}-4 c_{1} c_{3}}\right) /\left(2 c_{1}\right), \\
& c_{1}=-\beta^{2} \Gamma\left(3 v_{1}+1\right) \\
& \cdot\left\{[1-2 \varepsilon s+\varepsilon-k]\left(1+\beta_{0}^{2}\right)-2(\varepsilon-k) \beta^{-1} \beta_{0}\right\}, \\
& c_{2}=2 \Gamma\left(3 v_{1}+1\right) \\
& \cdot\left\{[1-(\varepsilon+k)]\left(1+\beta_{0}^{2}\right)+2(\varepsilon+k) \beta^{-1} \beta_{0}\right\}, \\
& c_{3}=3 \beta^{2} \Gamma\left[\varepsilon\left(3 v_{1}+1\right)+k\left(v_{1}+3\right)\right] \\
& \cdot\left\{(1-s)\left(1+\beta_{0}^{2}\right)-2 \beta^{-1} \beta_{0}\right\}+[1-s(\varepsilon+k)] \\
& \cdot\left\{\beta^{2}\left(8-3 \Gamma\left(v_{1}+3\right)\right)\left(1+\beta_{0}^{2}\right)\right. \\
& \left.-2 \beta^{-1} \beta_{0}\left[3\left(v_{2}+3\right)-\left(3 v_{2}+1\right)\left(2-\beta_{0}^{2}\right) \beta_{0}^{2}\right]\right\} .
\end{aligned}
$$

Similarly to Section 5 the rest of calculations including general form of characteristic Equations (8) and (10) coincide with the results presented in Section 4.

\section{Examples and Concluding Remarks}

For inhomogeneous disc with the parameters $n=2$, $\alpha=0.9, \quad \beta=0.93, \quad v_{1}=0.31, \quad v_{2}=0.3, \quad \varepsilon=1$, $\Gamma=0.99, s=0.99$ and $\sigma_{s 2} / E_{2}=0.01$, which loses its stability according to case (a) for $\beta_{0 *}=0.7148$ and $\omega_{*} / q_{2}=1.6825$ the values of critical radius $\beta_{0}^{*}$ of plastic zone D1p and relative critical rotation velocity $\omega^{*} / q_{2}$ are presented in Table 1.

Table 2 presents characteristic critical values obtained in terms of solution of characteristic Equation (10) for the disc with parameters $n=2, \beta=0.1, \quad v_{1}=0.4$, $v_{2}=0.3, \varepsilon=1, \quad \Gamma=1, s=0.8$ and $\sigma_{s 2} / E_{2}=0.01$ which loses its stability according to case (s2) for $\beta_{01 *}=\beta=0.1, \beta_{0 *}=0.7167$ and $\omega_{*} / q_{2}=1.6674$.

The same problem is solved for the disc with $n=2$, $\beta=0.5, \quad v_{1}=0.3, \quad v_{2}=0.2, \quad \varepsilon=1.2, \quad s=1.1 \quad$ and $\sigma_{s 2} / E_{2}=0.01$ whose instability develops according to (b) (Table 3) for $\Gamma=0.8, \beta_{0 *}=0.7106$ and $\omega_{*} / q_{2}=1.7310$ and according to (c) (Table 4) for $\Gamma=1$, $\beta_{01 *}=0.1889, \beta_{0 *}=0.7112$ and $\omega_{*} / q_{2}=1.7262$.

Table 1. Values of critical radius and relative critical velocity depending on $\delta$.

\begin{tabular}{cccccc}
\hline$\delta$ & $10^{-7}$ & $10^{-6}$ & $10^{-5}$ & $10^{-4}$ & $10^{-3}$ \\
\hline$\beta_{0}^{*}$ & 0.8999 & 0.8996 & 0.8967 & 0.8770 & 0.8031 \\
$\omega^{*} / q_{2}$ & 1.7228 & 1.7227 & 1.7223 & 1.7193 & 1.7046 \\
\hline
\end{tabular}

Table 2. Values of critical radius and relative critical velocity depending on $\delta$.

\begin{tabular}{cccccc}
\hline$\delta$ & $10^{-7}$ & $10^{-6}$ & $10^{-5}$ & $10^{-4}$ & $10^{-3}$ \\
\hline$\beta_{0}^{*}$ & 0.9932 & 0.9853 & 0.9682 & 0.9310 & 0.8474 \\
$\omega^{*} / q_{2}$ & 1.7146 & 1.7144 & 1.7138 & 1.7109 & 1.6984 \\
\hline
\end{tabular}

Table 3. Values of critical radius and relative critical velocity depending on $\delta$.

\begin{tabular}{cccccc}
\hline$\delta$ & $10^{-7}$ & $10^{-6}$ & $10^{-5}$ & $10^{-4}$ & $10^{-3}$ \\
\hline$\beta_{0}^{*}$ & 0.9927 & 0.9842 & 0.9658 & 0.9255 & 0.8339 \\
$\omega^{*} / q_{2}$ & 1.7733 & 1.7732 & 1.7725 & 1.7696 & 1.7567 \\
\hline
\end{tabular}


Table 4. Values of critical radius and relative critical velocity depending on $\delta$.

\begin{tabular}{rccccc}
\hline$\delta$ & $10^{-7}$ & $10^{-6}$ & $10^{-5}$ & $10^{-4}$ & $10^{-3}$ \\
\hline$\beta_{0}^{*}$ & 0.9926 & 0.9842 & 0.9658 & 0.9254 & 0.8338 \\
$\omega^{*} / q_{2}$ & 1.7779 & 1.7778 & 1.7769 & 1.7733 & 1.7572 \\
\hline
\end{tabular}

The relationships established between the value of boundary shape perturbation, location and type of perturbed elastoplastic boundary and rotation velocity of unstable continuous circular disc allow qualitative and quantitative conclusions to be made about peculiarities of the disc super-high-speed dynamics. The application of the obtained results enables us to forecast the development of unstable state and to calculate possible loss of stability and failure of bearing capacity of rotating discs.

It should be noted that basic equations of stability theory of spatial deformable bodies derived by linearization of nonlinear equations contain terms specified via the components of unperturbed ground state. This causes some difficulties in the problem on loss of stability, since a loading parameter associated with the critical efforts enters the basic equations. Application of the approximated approach presented in the paper for stability investigation of spatial elastic bodies simplifies the problem because both the perturbations $\sigma_{i j}^{\prime}$ satisfy the initial balance equations and the loading parameter is introduced into boundary conditions on the perturbed initial surface of the body. The loading parameter is determined by essentially more simple characteristic equations.

\section{REFERENCES}

[1] K. B. Bitseno and R. Grammel, "Technical Dynamics," Vol. 2, Gosudarstvennoe Izdatelstvo Tekhniko-Teoreticheskoy Literatury, Moscow and Leningrad, 1952.

[2] A. E. H. Love, "A Treatise on the Mathematical Theory of Elasticity,” Dover Publications, New York, 1927.

[3] S. P. Timoshenko and J. N. Goodier, "Theory of Elasticity," McGraw-Hill, New York, 1934.

[4] A. N. Guz and Yu. N. Nemish, "Method of Boundary Form Perturbation in the Mechanics of Continua," Vyshcha Shkola, Kiev, 1989.

[5] V. V. Sokolovsky, "Plasticity Theory," Vysshaya Shkola, Moscow, 1969.

[6] A. N. Guz and I. Yu. Babich, "Three-Dimensional Stability Theory of Deformable Bodies," Naukova Dumka, Kiev, 1985.
[7] A. Nadai, "Plasticity and Fracture of Solid Bodies," Vol. 1, Izdatelstvo Inostrannoy Literatury, Moscow, 1954.

[8] D. D. Ivlev, “Continuum Mechanics," Vol. 2, Phismatlit, Moscow, 2002.

[9] D. D. Ivlev, "On the Loss of Bearing Capacity of Rotating Discs, Close to Circular Ones," Izvestiya Akademii Nauk SSSR, Otdelenie Tekhnicheskikh Nauk, No. 1, 1957, pp. 141-144.

[10] D. V. Georgievskii, "Small Perturbations of an Undeformed State in Media with Yield Stress," Doklady Physics, Vol. 48, No. 10, 2003, pp. 590-593. doi:10.1134/1.1623545

[11] D. D. Ivlev and L. V. Yershov, "Perturbation Method in the Theory of Elastoplastic Body," Nauka, Moscow, 1978 .

[12] L. V. Yershov and D. D. Ivlev, "On the Stability Loss of Rotating Discs," Izvestiya Akademii Nauk SSSR, Otdelenie Tekhnicheskikh Nauk, No. 1, 1958, pp. 124-125.

[13] D. M. Lila, "Eccentric Form of Stability Loss of a Rotating Elastoplastic Disc," Reports of the National Academy of Sciences of Ukraine, No. 2, 2011, pp. 49-53.

[14] D. M. Lila and A. A. Martynyuk, "About the Stability Loss of a Rotating Elastoplastic Circular Disc," Reports of the National Academy of Sciences of Ukraine, No. 1, 2011, pp. 44-51.

[15] D. M. Lila and A. A. Martynyuk, "Development of Instability in a Rotating Elastoplastic Annular Disk," International Applied Mechanics, Vol. 48, No. 2, 2012, pp. 224 233.

[16] D. M. Lila, "On the Instability of Rotating Elastoplastic Stepped Annular Disc, Loaded over the Boundary in the Middle Plane," International Applied Mechanics (to be published)

[17] D. M. Lila and A. A. Martynyuk, "Stability Loss of Rotating Elastoplastic Discs of the Specific Form," Applied Mathematics, Vol. 2, No. 5, 2011, pp. 579-585. doi:10.4236/am.2011.25077

[18] I. V. Demianushko and I. A. Birger, "Stress Calculation of Rotating Discs," Mashinostroyeniye, Moscow, 1978.

[19] M. Mazière, J. Besson, S. Forest, B. Tanguy, H. Chalons and F. Vogel, "Overspeed Burst of Elastoviscoplastic Rotating Disks-Part I: Analytical and Numerical Stability Analyses," European Journal of Mechanics A/Solids, Vol. 28, No. 1, 2009, pp. 36-44. doi:10.1016/j.euromechsol.2008.07.008

[20] M. Mazière, J. Besson, S. Forest, B. Tanguy, H. Chalons and F. Vogel, "Overspeed Burst of Elastoviscoplastic Rotating Disks: Part II-Burst of a Superalloy Turbine Disk," European Journal of Mechanics A/Solids, Vol. 28, No. 3, 2009, pp. 428-432.

doi:10.1016/j.euromechsol.2008.10.002 